\title{
Glaciação de montanha em faixas orogenéticas Brasilianas no Cráton do São Francisco? Uma revisão e uma proposta
}

\author{
Guilherme Modesto Gonzaga' \& Detlef Hans-Gert Walde ${ }^{2}$
}

\begin{abstract}
Resumo Depósitos sedimentares neoproterozóicos, em parte glaciogênicos, relacionados a diversas faixas orogenéticas do Brasiliano em torno do Cráton do São Francisco, são aqui descritos e interpretados sob uma nova perspectiva. As ocorrências principais incluem: Faixa Brasília: diamictitos da Formação Samburá, diamictitos da base do Grupo Vazante (Formação. Santo Antonio do Bonito), diamictitos do topo do Grupo Vazante, diamictitos do Domo de Cristalina, diamictitos de Formosa, Padre Bernardo, Nova Roma e Minaçu, em Goiás e diamictitos de Campos Belos, em Tocantins; Faixa Araçuaí: Formação Nova Aurora, porção superior do Grupo Macaúbas, Formação Salinas, em Minas Gerais e Formação Salobro, na Bahia. Faixa Sergipano: diamictitos da Formação Palestina, em Sergipe; Faixa Rio Preto: diamictitos da Formação Canabravinha, na Bahia. Faixa Ribeira: rochas da parte superior da Bacia Andrelândia, em Minas Gerais. Este trabalho revisa esses depósitos tidos como glaciogênicos e relacionados ao evento glacial São Francisco por alguns, ou considerados não glaciogênicos por outros ou simplesmente não conhecidos ainda. Sugere-se que eles são produtos de uma glaciação de montanha, diretamente relacionada com a evolução tectônica das faixas de dobramento do Brasiliano. Diversas ocorrências de formação ferrífera e/ou fosforito são registradas em associação direta ou indireta a esses depósitos em parte glaciogênicos, atribuídos ao Ediacariano. Esse cenário geológico no Brasil é compatível com exemplos africanos.
\end{abstract}

Palavras-chave: glaciação, Brasiliano, Ediacariano, Cráton do São Francisco.

\begin{abstract}
Mountain glaciation in the Orogenic Brasiliano Belts of the São Francisco Craton? A review and a proposal. Neoproterozoic sedimentary deposits, partially glaciogenic, related to Brasiliano orogenic belts surrounding the São Francisco Craton are described and interpreted under a new perspective. The occurrences include: Brasília Belt: diamictites of the Samburá Formation, diamictites occurring at the base of the Vazante Group (Santo Antonio do Bonito Formation), diamictites at the top of the Vazante Group, diamictites of the Cristalina Dome, diamictites of Formosa, Padre Bernardo, Nova Roma and Minaçu localities in Goiás state, and diamicites of Campos Belos, in Tocantins state. Araçuaí Belt: Nova Aurora Formation, in the upper section of the Macaúbas Group, Salinas Formation in Minas Gerais, Salobro Formation in Bahia. Sergipano Belt: diamictites of the Palestina Formation, in Sergipe state. Rio Preto Belt: diamictites of the Canabravinha Formation, in Bahia state. Ribeira Belt: the upper section of the Andrelândia Basin, in Minas Gerais state. We review these glaciogenic deposits which had previously been either related to the São Francisco glacial event, not considered to be glaciogenic or simply not known. Further, we suggest the presence of a moutain related glacial event, directly related to the tectonic evolution of the different belts of the Brasiliano orogeny. Many occurrences of iron formation and/or phosphorite are registered in these sediments partially glaciogenic and attributed to the Ediacarian. This geological scenario in Brazil is compatible with african examples.
\end{abstract}

Key-words: glaciation, Brasiliano Orogeny, Ediacarian, São Francisco Craton.

INTRODUÇÃo Um evento glacial neoproterozóico de natureza continental conhecido como Glaciação São Francisco (Karfunkel et al. 2000) ou Glaciação Jequitaí (Couto \& Bez 1981), que ocorreu há 740 Ma no Cráton do São Francisco (anteriormente à orogênese Brasiliana, $\sim 630$ a $540 \mathrm{Ma}$ ), é bem documentado na literatura. Sua capa de carbonatos constituída pela Formação Sete Lagoas está datada em $740 \pm 22$ Ma (Babinski \& Kaufman 2003; Babinski et al. 2007). O segundo evento glacial do Neoproterózoico, conhecido na Faixa Paraguai, nos estados de Mato Grosso e Mato Grosso do Sul, não foi ainda correlacionado com outros depósitos glaciais da mesma idade no Brasil. O trabalho revisa vários depósitos glaciogênicos anteriormente atribuídos ao evento glacial São Francisco, ou não tidos como glaciogênicos, ou simplesmente ainda não conhecidos. Gonzaga (2001) sugeriu, baseado na observação de um grande número dessas ocorrências, a possível existência de um evento glacial de altitude, diretamente relacionado com a evolução tectônica das diferentes faixas do Brasíliano. Apontou a origem desses depósitos por retrabalhamento gravitacional de sedimentos, como também demonstrou, localmente, a presença de fosforito e/ou formação ferrífera típica do final do Neoproterozóico. Esse segun- 
do evento glacial Neoproterozóico no estado de Minas Gerais foi denominado Glaciação Samburá (Gonzaga 2001). Depósitos glaciais do final do Neoproterozóico não estão restritos a regiões ao redor do Cráton do São Francisco, mas também ocorrem nos estados de São Paulo e Paraná, bem como em outras regiões do Brasil (Gonzaga 2001, Walde \& Gonzaga 2005a, b).

\section{EXEMPLOS SELECIONADOS DE DEPÓSITOS GLACIOGÊNICOS DO EDIACARIANO}

Faixa Brasília Na Faixa Brasília do estado de Minas Gerais ocorrem diamictitos nas regiões de São Roque de Minas, Presidente Olegário, Lagamar, Lagoa Formosa e Campos Altos, considerados equivalentes à Formação Samburá. Também são conhecidos diamictitos pertencentes à Formação Santo Antônio do Bonito (Dardenne 2001) na base do Grupo Vazante, como também a possíveis depósitos glaciogênicos próximo ao topo do Grupo Vazante, na base da Formação Lapa (Azmy et al. 2006). No estado de Goiás são conhecidos diamictitos na região de Cristalina, Formosa, Padre Bernardo, Nova Roma e Minaçú. No estado de Tocantins são conhecidos diamictitos em Campos Belos. Com exceção do Grupo Vazante, todas as outras unidades litoestratigráficas foram interpretadas até por volta do ano 2000 como sedimentos em parte glaciogênicos, relacionados com a única glaciação neoproterozóica conhecida na região (Glaciação São Francisco, Karfunkel et al. 2000).

Barbosa et al. (1970) citaram a ocorrência de conglomerados intraformacionais (Fácies Molássica Samburá) na estrada entre Presidente Olegário e Lagamar e nas proximidades de Campos Altos. Esses depósitos sedimentares foram posicionados, segundo os autores, sobre a Formação Sete Lagoas (Grupo Bambuí) e correlacionados com os "Conglomerados" Samburá (Branco 1956) na região da Serra da Canastra (São Roque de Minas). Dardenne et al. (1978), Gonzaga \& Tompkins (1991) e outros consideraram os depósitos como glaciogênicos e relacionados com o evento glacial São Francisco (ou Glaciação Jequitaí de Couto \& Bez 1981), de distribuição regional na base do Grupo Bambuí. Essa hipótese conflita com o nítido posicionamento do "Conglomerado Samburá" próximo ao topo da Formação Sete Lagoas (Grupo Bambuí), de acordo com Castro \& Dardenne (1996) e outros. Alkmim et al. (2001) consideraram os conglomerados Samburá como uma formação do Grupo Bambuí, posicionada sobre a Formação Sete Lagoas e sotoposta à Formação Serra de Santa Helena (Fig. 1).

A Formação Samburá (Alkmim et al. 2001) em São Roque de Minas, região da Serra da Canastra apresenta, segundo Gonzaga \& Tompkins (1991), conglomerados polimíticos, diamictitos e pelitos com clastos caídos (dropstones). A matriz dos diamictitos e também alguns níveis pelíticos freqüentemente enriquecidos em fósforo, podendo atingir teores de $20 \% \mathrm{P}_{2} \mathrm{O}_{5}$ em superfície (lateritas) e teores de 5\% na matriz dos diamictitos e nas rochas pelíticas.

Os diamictitos apresentam clastos (de grânulos a matacões) dispersos em uma matriz síltico-argilosa, algumas vezes arenosa. Os clastos são principalmente de quartzito e quartzo, ocorrendo também gnaisse, filito, carbonato e chert vermelho. Blocos e matacões pentagonais (flat-iron) são freqüentes (Gonzaga \& Tompkins 1991). Os sedimentos da Formação Samburá foram apontados, na região da Serra da Canastra, como glaciogênicos e considerados possivelmente diamantíferos (Branco 1956, Gonzaga \& Tompkins 1991).

Seer et al. (1987) identificaram e descreveram diamictitos com matriz argilosa a síltica, que gradam para ardósias, associados ao Grupo Bambuí na região de Lagoa Formosa, Minas Gerais. Os clastos presentes nos diamictitos (15 a $20 \mathrm{~cm}$ de diâmetro maior, podendo atingir 1 metro) são subangulares a subarredondados e constituídos principalmente por metassiltito e metaarenito e ocasionalmente por ardósia e arcósio. Gnaisse, granito, metaconglomerado e jaspelito são raros. Os autores também identificaram jaspelitos em contato concordante com ritmitos. Os jaspelitos apresentam lâminas de chert e hematita intercaladas com lâminas de grãos detríticos. Gonzaga (2001) interpretou essa seqüência como equivalente à Formação Samburá.

Uma seqüência metassedimentar neoproterozóica na região de Fortaleza de Minas e Itaú de Minas, constituída de mármores, clorita e sericita-filitos, quartzitos, além de rochas fosfatadas e formação ferrífera (BIF) foi descrita por Fernandes et al. (2000). Segundo os autores, a presença da formação ferrífera é indicativa de um suprimento pobre em terrígenos durante um evento glacial e da presença de águas com bastante oxigênio que promoveram a oxidação e precipitação de ferro. Os fosfatos (marinhos) seriam formados pela mudança das condições glaciais para não glaciais por meio da redução da solubilidade dos fosfatos em águas quentes. $\mathrm{O}$ teor de $\mathrm{P}_{2} \mathrm{O}_{5}$ chega a atingir 25\% (Carvalho et al. 2000). Essa seqüência metassedimentar no extremo sul da Faixa Brasília, é provavelmente correlacionável com a seqüência metassedimentar estudada por Seer et al. (1987) na região de Lagoa Formosa que, segundo Gonzaga (2001), seria equivalente à Formação Samburá.

A ocorrência de zircões detríticos com idade máxima de $670 \mathrm{Ma}$ (Dardenne et al. 2003) na Formação Samburá aponta uma idade ediacariana e descarta o evento glacial São Francisco ( 750 Ma). É importante ressaltar que Castro \& Dardenne (1996) interpretaram uma proveniência dos sedimentos a partir de oeste, aporte esse relacionado a uma bacia do tipo foreland. Gonzaga (2001) apontou a Formação Samburá do Grupo Bambuí, como composta por sedimentos em parte glaciogênicos, relacionados à glaciação de altitude ocorrida durante o Brasiliano e correlacionável com os diamictitos da base do Grupo Vazante (Fig. 2).

Gonzaga \& Tompkins (1991) descreveram diamictitos com matriz fosfatada na região da cabeceira do rio Santo Antônio do Bonito, no município de Coromandel, MG. Posteriormente esse diamictito foi posicionado por Souza (1997) e Dardenne et al. (1997) na base do Grupo Vazante (Formação Retiro e, posteriormente, Formação Santo Antonio do Bonito, Dardenne 2001). Dardenne (2000) indica proveniência a partir de 


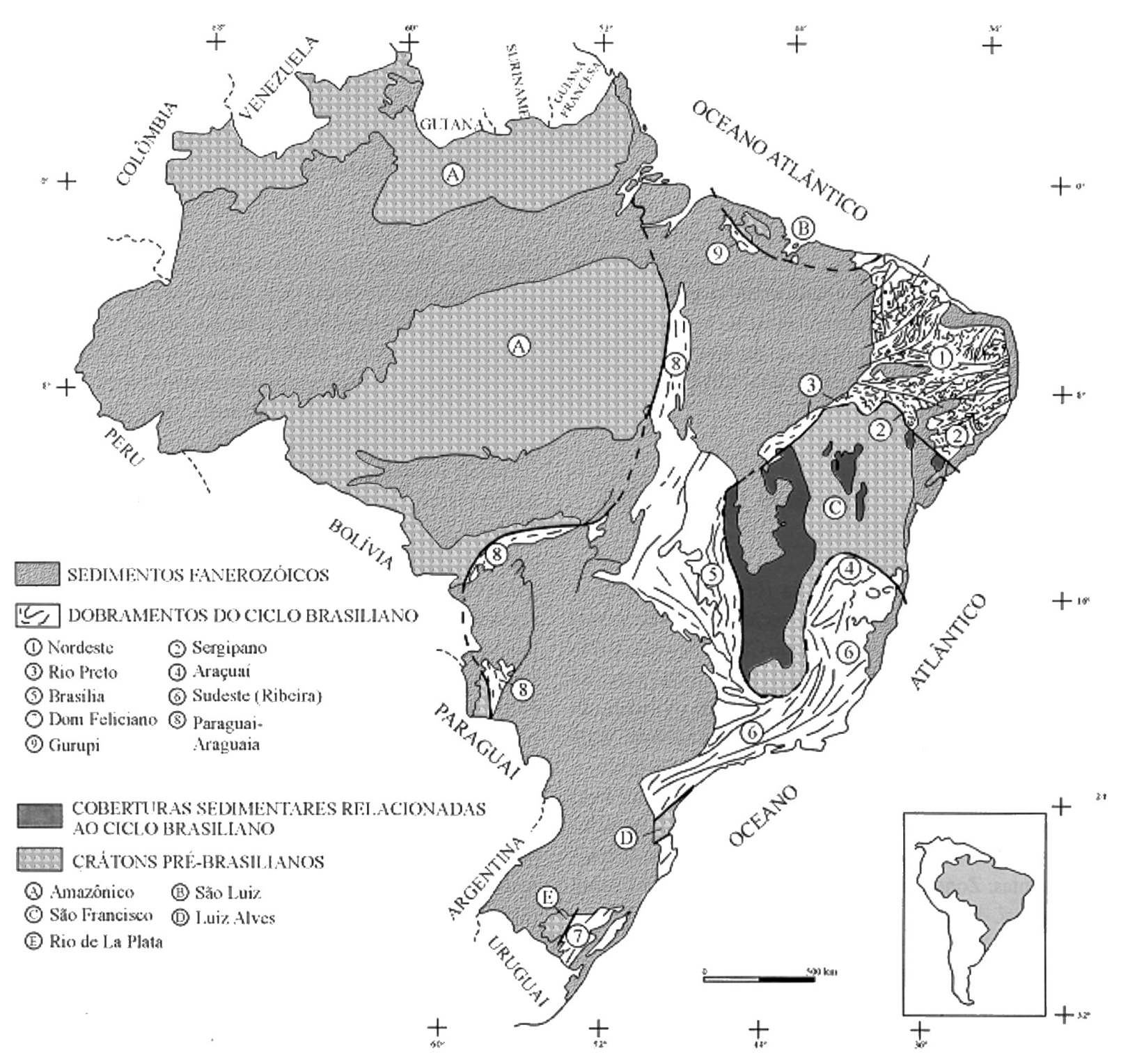

Figura 1 - Faixas de dobramentos do Ciclo Brasiliano e crátons correlatos (adaptado de Schobbenhaus \& Campos 1984 e Dardenne 2000).

oeste para leste (Fig. 3), o que sugere que houve um retrabalhamento do Grupo Ibiá. Esse fato sugere que os diamictitos, possivelmente glaciogênicos, são posteriores à Glaciação São Francisco, evento esse que teria originado a Formação Ibiá.

Azmy et al. (2000) obtiveram valores entre 0,706 e $0,7075\left({ }^{87} \mathrm{Sr} /{ }^{86} \mathrm{Sr}\right)$ em dolomitos do Grupo Vazante, comparáveis à assinatura isotópica $(\mathrm{Sr})$ dos mares do Ediacariano. Fosforito da base do Grupo Vazante, datado por Babinski et al. (2002), apresentou idade mínima de $690 \pm 76 \mathrm{Ma}$ e razão ${ }^{87} \mathrm{Sr} /{ }^{86} \mathrm{Sr}$ de 0,7077 , coerente com valores da água do mar do final do Neoproterozóico (730-600 Ma). Misi et al. (2007), com base em valores de $\delta^{13} \mathrm{C}$, correlacionaram a Formação Pamplona do Grupo Vazante com o Grupo Corumbá, no Mato Grosso do Sul, que é do Ediacariano. Kaufman et al. (2005) descrevem a presença de superfície erosional no topo do Grupo Vazante, rochas carbonático-pelíticas com clastos caídos, diamictitos e, localmente, formação ferrífera. Os autores indicam a ocorrência de glendonita, mineral mais comumente formado entre $-1,9$ e $7^{\circ} \mathrm{C}$, cuja presença já foi reportada em outros depósitos glaciais do Neoproterozóico.

Cukrov (1999) reafirma a opinião de que os diamictitos possivelmente glaciogênicos do Domo de Cristalina, em Goiás, correspondem à Glaciação São Francisco. É importante observar que Trompette (1994) já havia proposto a correlação dessas ocorrências com a seqüência descrita por Seer et al. (1987) na região de Lagoa Formosa (Minas Gerais), bem como com os diamictitos na região de Formosa (Goiás). Pimentel et al. (2002), com base em datações de zircões detríticos (U-Pb SHIMP), apontam uma proveniência para os sedimentos glaciogênicos de Cristalina preferencialmente a partir de NW do Cráton do São Francisco, com contribuição do Grupo Araí. Essa direção de transporte 


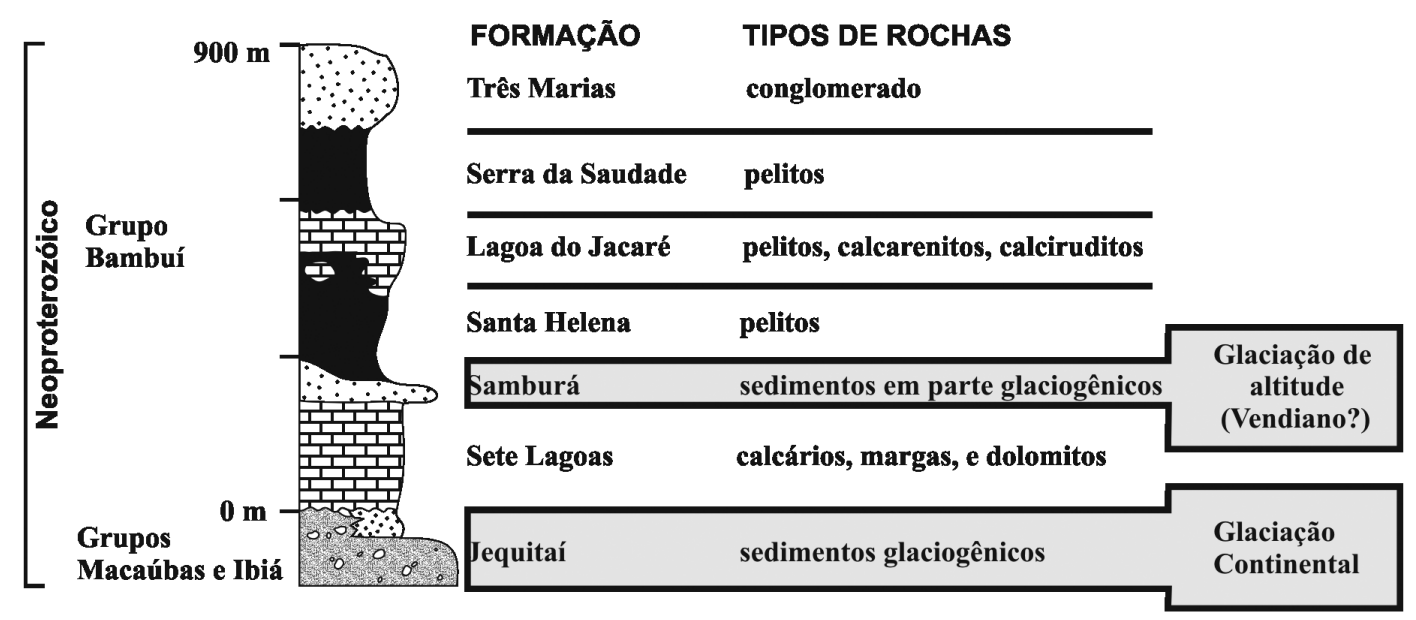

Figura 2 - Posicionamento das glaciações Jequitaí e Samburá em relação ao Grupo Bambui (adaptada de Alkmim et al. 2001 e Gonzaga 2001)

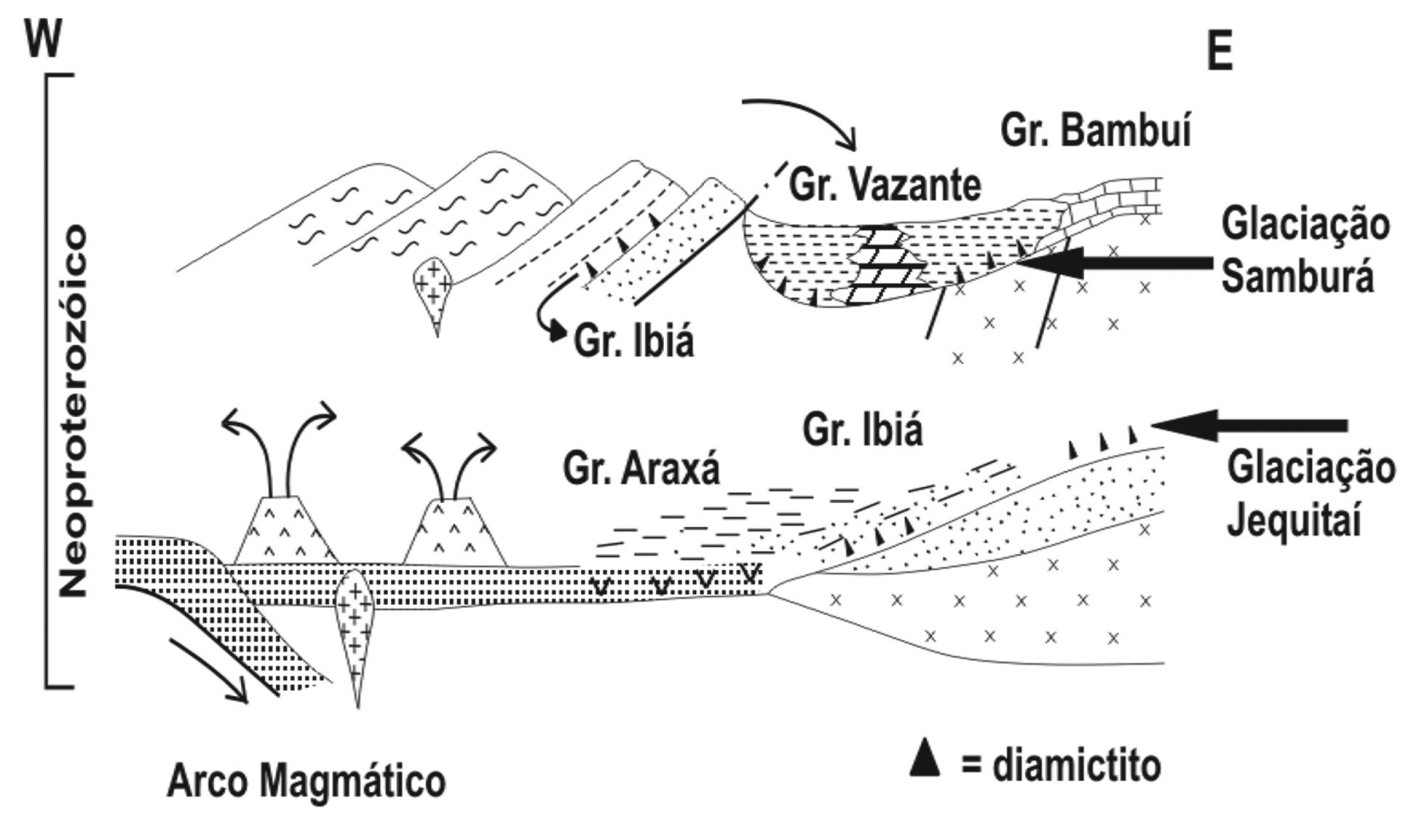

Figura 3 - Modelo esquemático mostrando a natureza continental da glaciação Jequitaí e a natureza da glaciação Samburá como de montanha (adaptada de Dardenne 2000 e Gonzaga 2001)

não é a esperada para os sedimentos da Glaciação São Francisco (Grupo Ibiá), que são oriundos de nordeste/ leste. Dardenne et al. (2003) admitem que os depósitos glaciogênicos do Domo de Cristalina podem ser algo diferente do esperado. Lima et al. (2003b) comparam os sedimentos de Cristalina com os do Grupo Ibiá (Tab. 1). Na opinião dos autores do presente trabalho são bastante claras as diferenças entre as duas unidades, reforçando a idéia de que os diamictitos (glaciogênicos?) de Cristalina estão relacionados a um evento glacial de montanha que teria ocorrido durante o Ediacariano.

Os diamictitos possivelmente glaciogênicos de
Formosa (Goiás) foram correlacionados por Trompette (1994) com rochas atualmente interpretadas como pertencentes à Formação Samburá, na região de Lagoa Formosa, em Minas Gerais, que originalmente foram descritos por Seer et al. (1987).

Uma correlação do diamictito Samburá em Minas Gerais com o diamictito de Formosa já foi sugerida por Gonzaga \& Tompkins (1991), com base na presença de fosforito, tanto nos diamictitos Samburá (Gonzaga \& Tompkins 1991), como nos diamictitos de Formosa (Dardenne et al. 1978).

Na região de Padre Bernardo (Goiás) ocorre 
Tabela 1 - Comparação entre os diamictitos do Grupo Ibiá (Fm. Cubatão) e de Cristalina, GO (adaptada de Lima et al. 2003b).

\begin{tabular}{|c|c|c|}
\hline & Sedimentos glaciogênicos de Cristalina - GO & Grupo Ibiá (Guarda-Mor - MG) \\
\hline Espessura (m) & $\sim 100$ & 550 a 1.000 \\
\hline Composição dos clastos & $\begin{array}{l}\text { Quartzito (predominantemente), metassílticos } \\
\text { brancos, quartzo, filitos hematíticos (com cores } \\
\text { vermelho escuro a preto) e carbonatos. }\end{array}$ & $\begin{array}{l}\text { Carbonatos (mais freqüentes). Quartzitos, } \\
\text { granitos (alguns com textura pegmatóide), } \\
\text { gnaisses, micaxistos, quartzo, filitos. }\end{array}$ \\
\hline Dimensão dos clastos & $\begin{array}{l}\text { Ocorrem alguns pouquíssimos matacões de } \\
\text { quartzito, que alcançaram até } 40 \mathrm{~cm} \text {, no entanto, } \\
\text { a grande maioria se encontra abaixo de } 5 \mathrm{~cm} \\
\text { (seixos). Ocorrem, ainda, muitos grânulos de } \\
\text { quartzo e carbonato. }\end{array}$ & $\begin{array}{l}\text { Grande maioria situada entre } 3 \text { e } 10 \mathrm{~cm} \text {, exceção } \\
\text { feita ao carbonato, normalmente, inferiores } \\
\text { a } 2 \mathrm{~cm} \text {, e a alguns matacões de granotóides, } \\
\text { micaxistos e quartzitos, que às vezes alcançam } \\
1 \mathrm{~m} \text {. }\end{array}$ \\
\hline Forma dos clastos & $\begin{array}{l}\text { Normalmente angulosos a subangulosos, pouco } \\
\text { deformados. }\end{array}$ & $\begin{array}{l}\text { Clastos angulosos a subarredondados, } \\
\text { deformados e estirados. }\end{array}$ \\
\hline Composição da matriz & Silto-arenosa. & $\begin{array}{l}\text { Predominantemente pelítica, localmente } \\
\text { arenosa. }\end{array}$ \\
\hline Contato basal & $\begin{array}{l}\text { Discordância angular e erosiva com o Grupo } \\
\text { Paranoá. }\end{array}$ & $\begin{array}{l}\text { Discordância angular e erosiva com o Grupo } \\
\text { Canastra. }\end{array}$ \\
\hline Contato de topo & $\begin{array}{l}\text { Contato tectônico, filitos do Grupo Canastra } \\
\text { cavalgam a Formação Jequitaí. }\end{array}$ & $\begin{array}{l}\text { Contato gradacional para os metarritmitos da } \\
\text { Formação Rio Verde. }\end{array}$ \\
\hline $\begin{array}{l}\text { Estruturas sedimentares, } \\
\text { estratificações }\end{array}$ & $\begin{array}{l}\text { Camadas de diamictitos com intercalações } \\
\text { de ritmitos. Seixos facetados e estriados são } \\
\text { descritos por Faria (1985). }\end{array}$ & $\begin{array}{l}\text { Sugestão de camadas superpostas de diamictitos } \\
\text { com variações na matriz, tamanho e composição } \\
\text { dos clastos. }\end{array}$ \\
\hline $\begin{array}{l}\text { Elementos estruturais e } \\
\text { grau de deformação }\end{array}$ & $\begin{array}{l}\text { Baixo grau de deformação, contendo uma } \\
\text { xistosidade incipiente e alguns poucos clastos } \\
\text { achatados. Fraturas e veios de quartzo estão } \\
\text { praticamente ausentes. }\end{array}$ & $\begin{array}{l}\text { Xistosidade proeminente, ora intersectada por } \\
\text { clivagem de crenulação }\left(\mathrm{S}_{\mathrm{n}+1}\right) \text { e muitos seixos } \\
\text { alongados e achatados, definindo uma lineação } \\
\text { de estiramento. }\end{array}$ \\
\hline
\end{tabular}

diamictitos fosfatados (Dardenne et al. 1978) também relatado pelo projeto RADAMBRASIL (1981). Esse relatório indica ainda a presença de itabirito (quartzito magnetítico-hematítico).

Os diamictitos das regiões de Nova Roma e Minaçu, bem como de Campos Belos (Tocantins) foram descritos como glaciogênicos e eventuais portadores de fosforito (Dardenne et al. 1978).

Faixa Araçuaí A figura 4 mostra o Grupo Macaúbas dividido na região de Porteirinha (Minas Gerais) em dois setores, ocidental e oriental. O setor ocidental inicia-se com a Formação Serra do Catuni, formada por clássicos depósitos glaciogênicos de natureza continental, comparáveis à Formação Jequitaí (Karfunkel et al. 2000, Karfunkel et al. 2001 e outros). O setor oriental do Grupo Macaúbas inicia-se com a Formação Nova Aurora, que mostra evidências de retrabalhamento gravitacional (Uhlein 1991). Nessa formação são conhecidos importantes depósitos de formação ferrífera e também algumas ocorrências de fosforito (Oliveira et al. 1997 e outros). Essa formação grada lateralmente para a Formação Chapada Acauã, que representa depósitos turbidíticos (Uhlein 1991, Oliveira et al. 1997). Tam- bém são conhecidas algumas ocorrências de formação ferrífera. A Formação Chapada Acauã grada para a Formação Salinas que é constituída por depósitos turbidíticos mais distais (Oliveira et al. 1997 e outros). Lima et al. (2003a) apresentam datações (U-Pb) de zircões detríticos da Formação Salinas com valores que limitam a idade máxima da sedimentação em 568 Ma.

A Formação Salinas representa sedimentação tardi-orogênica depositada no intervalo 568-500 Ma (Lima et al. 2003a). Além disso, outras evidências tais como ocorrências de formação ferrífera e fosforito reforçam a hipótese de que a seqüência Macaúbas oriental (Fig. 4) pode estar relacionada a um segundo evento glacial Neoproterozóico. Fácies ferrífera na Formação Nova Aurora, na região de Porteirinha, revelou um teor médio de fósforo de $0,33 \% \mathrm{P}_{2} \mathrm{O}_{5}$, obtido por meio de média ponderada em 1.364 amostras (Vilela et al. 1978). Também ocorre apatita disseminada e em níveis contínuos com espessura média de $1 \mathrm{~mm}$ (Oliveira et al. 1997). O sincronismo com o Evento Tectônico Brasiliano sugere que esse conjunto de rochas metassedimentares do setor oriental (Fig. 4) estaria diretamente relacionado ao desenvolvimento da Faixa Araçuaí. Seria uma glaciação de montanha e não uma glaciação 

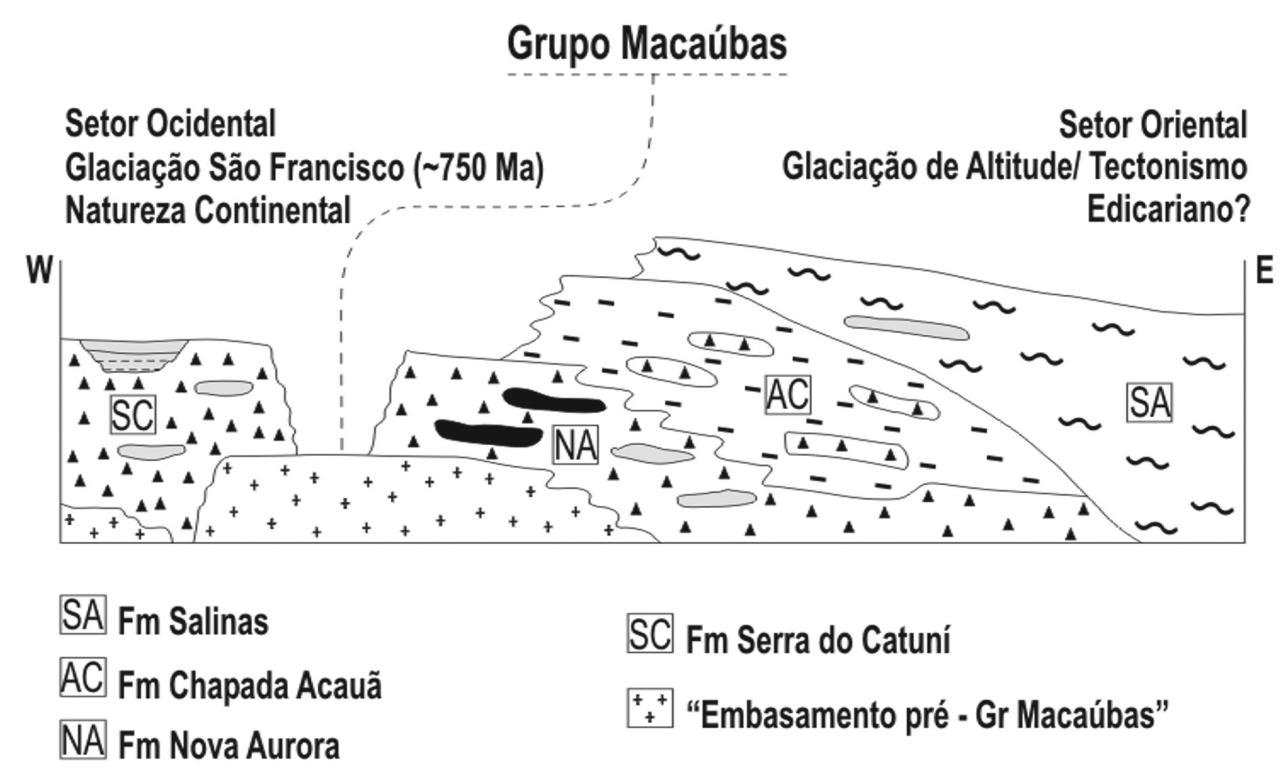

SC Fm Serra do Catuní

"Embasamento pré - Gr Macaúbas"

Figura 4 - Esquema estratigráfico do Grupo Macaúbas na região de Porteirinha - Minas Gerais. (adaptada de Oliveira et al. 1997 e Walde \& Gonzaga 2005c).

continental, como é o caso do evento glacial observado no setor Macaúbas ocidental.

Esse segundo evento glacial, embora nunca tenha sido sugerido anteriormente na Faixa Araçuaí no estado de Minas Gerais, pode justificar a forte analogia que alguns autores (Walde 1986, Hoppe et al. 1987) fizeram como o evento glacial Ediacariano na Faixa Paraguai, na região de Corumbá-MS. Trompette (1994) mencionou que os depósitos de formação ferrífera, na África são mais jovens do que a idade admitida para a formação ferrífera de Porteirinha (Grupo Macaúbas).

$\mathrm{Na}$ Bahia, a presença de diamantes em conglomerados da Formação Salobro (Grupo Rio Pardo) na região de Canavieiras, foi comprovada por Derby (Baptista et al. 1984). Segundo Leonardos (1940), D. Guimarães sugeriu uma origem glacial para os conglomerados da Formação Salobro (Neoproterozóico) na bacia do rio Pardo. É bastante provável que os conglomerados flúvio-glaciais da Formação Salobro sejam produtos de glaciação de montanha do Cambriano, que seria um prolongamento da atividade glacial do Neoproterozóico. Essa situação foi reportada na África por Evans (2000b).

Faixa Sergipana Na Faixa Sergipana são conhecidas duas unidades litoestratigráficas neoproterozóicas portadoras de diamictitos. A mais jovem delas (Formação Palestina) apresenta zircões com idade mínima de 570 Ma e está relacionada a uma bacia de foreland formada durante a colisão do Maciço Pernambuco-Alagoas com o Cráton do São Francisco, durante o Brasíliano (Oliveira et al. 2005 a, b). Oliveira et al. (2005b), na apresentação oral do trabalho, sugeriram uma origem glaciogênica para os diamictitos da Formação Palestina.
Faixa Rio Preto A Formação Canabravinha, base do Grupo Bambuí, apresenta diamictitos com clastos caídos e freqüentemente facetados, intercalação de itabirito (formação ferrífera) e turbiditos (Andrade Filho et al. 1994). Conforme pode ser visto na figura 5 de Uhlein et al. (2004), a proveniência dos sedimentos é de oeste e não se observa a presença de sedimentos carbonáticos correlacionáveis à Formação Sete Lagoas. Essa situação geológica não é compatível com o evento glacial São Francisco comumente referido na bibliografia, mas sim com uma glaciação de montanha desenvolvida durante o evento Brasíliano.

Faixa Ribeira No topo da bacia Andrelândia (Minas Gerais) ocorrem diamictitos e seqüência pelítica com clastos caídos de natureza glaciogênica (Trow et al. 2000) que, localmente, podem apresentar uma alta proporção de apatita (Ribeiro et al. 2003). Heilbron et al. (2004) sugerem uma correlação temporal desses depósitos com o evento Brasiliano.

ASPECTOS ECONÔMICOS A presença de fosforito e/ou formação ferrífera pode colaborar na correlação dos eventos glaciais de altitude do Ediacariano (Gonzaga 2001). Conforme foi apresentado nesse trabalho, fosforitos na Faixa Brasília encontram-se associados a rochas da Formação Samburá e da base do Grupo Vazante em Minas Gerais, a diamictitos de Formosa, Padre Bernardo, Nova Roma e Minaçu em Goiás, bem como a diamictitos de Campos Belos (Tocantins). Fosforitos também ocorrem na porção superior do Grupo Macaúbas (Faixa Araçuaí) e na porção superior da Bacia Andrelândia, na Faixa Ribeira (Minas Gerais).

Formação ferrífera encontra-se associada a sedimentos equivalentes à Formação Samburá em Lagoa Formosa e Fortaleza de Minas / Itaú de Minas. Ainda 


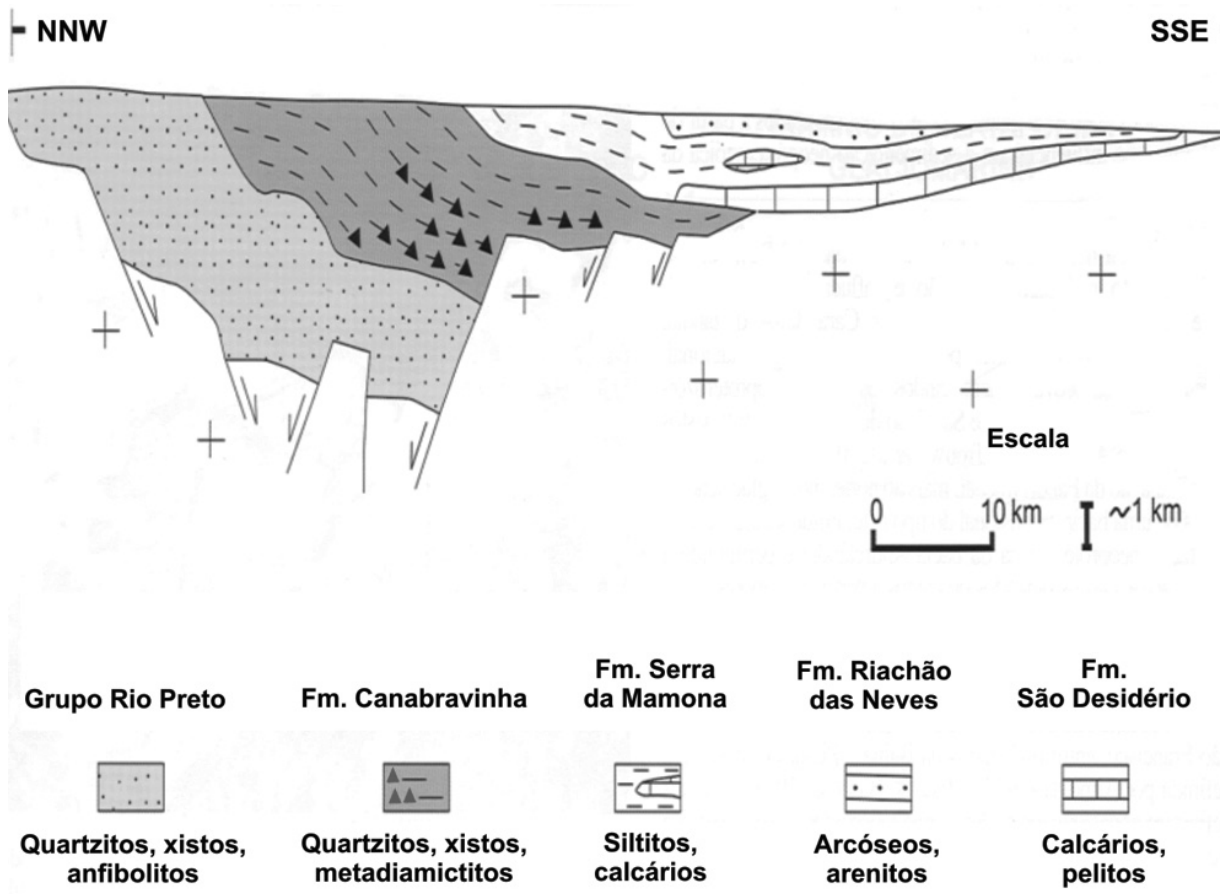

Figura 5 - Esquema estratigráfico na Faixa Rio Preto (seg. Uhlein et al. 2004).

na Faixa Brasília foi descrita a presença de formação ferrífera no topo do Grupo Vazante. Na Faixa Araçuaí, em Minas Gerais, sua ocorrência é bem conhecida na porção superior do Grupo Macaúbas, região de Porteirinha. Em Goiás (Faixa Brasília) é conhecida a presença de formação ferrífera em Padre Bernardo. Também ocorre no diamictito da Formação Canabravinha, na Bahia (Faixa Rio Preto).

Ocorrências de diamantes encontram-se diretamente associadas a diamictitos da Formação Samburá, em São Roque de Minas (Branco 1956; Gonzaga \& Tompkins 1991), a diamictitos da Formação Santo Antônio do Bonito (Grupo Vazante, em Coromandel, Minas Gerais, Gonzaga \& Tompkins 1991; Gonzaga 2001), a diamictitos da porção superior do Grupo Macaúbas em Minas Gerais (Walde \& Gonzaga, 2005c) e a conglomerados da Formação Salobro na Bahia (Derby 1906). Os garimpos de diamante são resultantes de retrabalhamento desses depósitos por diversos ciclos fluviais, em diferentes épocas.

Assim, os eventos glaciais ocorridos durante o Brasiliano seriam indicativos da presença de fosforito e/ou formação ferrífera. Depósitos econômicos são encontrados em Rocinha (fosforito) e em Urucum (ferro).

DISCUSSÃO A interpretação plausível baseada nas informações e hipóteses apresentadas sugere que depósitos glaciogênicos descontínuos, não restritos a um simples horizonte e freqüentemente de natureza gláciomarinha, apresentando intenso retrabalhamento gravitacional (turbiditos), com níveis de formação ferrífera e fosforito são produtos de glaciação de montanha do Ediacariano e não estão relacionados ao evento glacial São Francisco ocorrido há 750 Ma.
Uma diferenciação tectônica criando áreas positivas (blocos de soerguimento) e negativas (bacias de subsidência) proporcionou condições favoráveis ao desenvolvimento de glaciação de montanha durante o evento Brasíliano. Esse modelo de diferenciação tectônica foi apontado por Schermerhorn (1983). A partir dos blocos com soerguimento, sedimentos glaciogênicos foram transportados para as bacias de subsidência. Localmente geleiras chegaram até o nível do mar depositando sedimentos, alternando com outros sem influência glacial. O retrabalhamento gravitacional de sedimentos glaciais e não glaciais pode ser observado, em diferentes depósitos, nas diferentes faixas do Brasíliano.

Diamictitos possivelmente glaciogênicos, intercalados em espessos horizontes carbonáticos, ocorrem no Grupo Vazante (Faixa Brasília) bem como abaixo e acima da Formação Araras, na Faixa Paraguai, em Mato Grosso (Figueiredo et al. 2004).

Novas datações radiométricas têm indicado que esses tipos de depósitos são do final do Neoproterozóico, apresentando idades do Ediacariano. Uma importante conseqüência geológica desse modelo proposto inicialmente por Gonzaga (2001) e desenvolvido por Walde \& Gonzaga (2005a,b,c,d), bem como por Gonzaga (2005), é que a deposição dos grupos Bambuí e Vazante teve início com depósitos glaciogênicos de montanha, durante o evento Brasíliano e não está relacionada com a Glaciação São Francisco ocorrida ha $\sim 750 \mathrm{Ma}$. Este modelo é compatível com o descrito para o oeste africano por Caby (1987 apud Trompette 1994) e por Bertrand-Sarfati et al. (1987).

A questão levantada por Kaufman (1998) relativa à possível presença de dois eventos glaciais neoproterozóicos ocorridos em Minas Gerais pode ser res- 
pondida por meio desse modelo. Também é compatível com a amplamente divulgada presença de dois eventos glaciais do Neoproterozóico na África (740 e 600 Ma), conforme as idades obtidas por Evans (2000a). Frimmel \& Fölling (2000), usando datações radiométricas, concordam com essas idades para as glaciações e correlacionam com os períodos glaciais do Sturtiano e Ediacariano. Condições glaciais do final do Neoproterozóico podem localmente persistirem até o Cambriano, como parece ocorrer no oeste da África (Evans 2000b).

Agradecimentos Os autores agradecem as criticas e sugestões construtivas de diversos colegas, que sem dúvida alguma contribuíram para o aprimoramento do trabalho. Também agradecem aos revisores da Revista Brasileira de Geociências.

\section{Referências}

Alkmim F.F., Marshak S., Fonseca M.A. 2001. Assembling west Gondwana in the Neoproterozoic: clues from the São Francisco Craton region, Brazil. Geology. 29:319322.

Andrade Filho E.L., Neves J.P., Guimarães J.T. 1994. Santa Rita de Cássia (Folha SC.23-Z-C). Formosa do Rio Preto (Folha SC.23-Y-D). PLGB.CPRM. $50 \mathrm{p}$.

Azmy K., Veizer J., Misi A., De Oliveira T., Dardenne M.A., Sanches A. 2000. Dolomitization and isotope stratigraphy of the Vazante Formation, São Francisco Basin, Brazil. In: International Geological Congress. 31, Rio de Janeiro, Brazil, Abstracts, CD-ROOM.

Azmy K., Kaufman A.J., Misi A. \& Oliveira T.F. de. 2006. Isotope stratigraphy of the Lapa Formation, São Francisco Basin, Brazil: Implications for Late Neoproterozoic glacial events in South America. Precambrian Res., 149:231-248.

Babinski M., Sanches A.L., Misi A., Ruiz I.R. 2002. Datação $\mathrm{Pb}-\mathrm{Pb}$ do fosforito de Lagamar, MG. In: Congresso Brasileiro de Geologia, 41, Anais, p. 488.

Babinski M. \& Kaufman A.J. 2003. First direct dating of a Neoproterozoic post-glacial cap carbonate. In: South American Symposium on Isotope Geology. 4, Short Papers, p. 321.

Babinski M., Vieira L.C., Trindade R.I.F. 2007. Direct dating of the Sete Lagoas cap carbonate (Bambuí Group, Brazil) and implications for the Neoproteozoic glacial events. Terra Nova, 19:401-406.

Baptista M.B., Braun O.P.G., Campos D.A. 1984. Léxico Estratigráfico do Brasil. Brasília, DNPM. 222 p.

Barbosa O., Braun O.P.G., Dyer R.C., Cunha C.A.B.R. 1970. Geologia de região do Triângulo Mineiro. Rio de Janeiro. DNPM/DFPM, Boletim 136, 140 p.

Bertrand-Sarfati J., Moussine-Pouchkine A., Caby R. 1987. Les correlations du Protérozóïque au Cambrien en Afrique de L'Ouest: Nouvelle Interprétation Géodynamique. France Soc. Géol. Bull., 8.III:855-865.

Branco J.J.R. 1956. Conglomerado do Samburá, Minas Gerais. An. Acad. Bras. Ciênc., 28:295-301.

Caby R. 1987. The Pan-African belt of West Africa from the Sahara desert to the Gulf of Benin. In: Schaer J.P. \& J. Rodeers (eds). The Anatomy of Mountain Ranges. Princeton Univ. Press, p. 129-170.

Carvalho S.G., Brenner T.L., Zanardo A., Waring M.H., Feola J.L. 2000. Proterozoic phosphat deposits near Fortaleza de Minas, southwest Minas Gerais. Brazil. In: Intern. Geol. Cong., 31, Rio de Janeiro, Brazil, Abstracts, CD-ROM.
Castro P.T.A. \& Dardenne M.A. 1996. O Conglomerado Samburá (Grupo Bambuí, Neoproterozóico) e rochas sedimentares associadas no flanco leste da Serra da Pimenta, SW de Minas Gerais: um sistema de fan-delta. Geonomos, 3:35-41.

Couto J.G.P \& Bez L. 1981. A Glaciação Jequitaí: Um guia estratigráfico para o Pré-Cambriano Superior no Brasil. Rev. Bras. Geoc., 11:17-21.

Cukrov, N. 1999. A Glaciação Neoproterozóica na porção sul do Cráton do São Francisco e suas litofácies nas regiões de Jequitaí-MG e Cristalina-GO. Dissertação de Mestrado $\mathrm{n}^{\circ}$ 141. Instituto de Geociências. Universidade de Brasília.

Dardenne M.A. 2000. The Brazilian Fold Belt, In: Cordani U.G., Milani E.J., Thomaz Filho A., Campos D.A. (eds). Tectonic Evolution of South America. International Geological Congress. 30 ${ }^{\text {st }}$, Rio de Janeiro, Brazil, p. 231-363.

Dardenne M.A. 2001. Lithostratigraphic sedimentary sequences of the Vazante Group. In: Misi A. \& Teixeira J.B.G. (eds.) Proterozoic Base Metal Deposits of Africa and South America. IGCP 450, $1^{\text {st }}$, Belo Horizonte and Paracatu, Brazil, Field Workshop, p. 48-50.

Dardenne M.A., Faria A., Magalhães L., Soares L.A. 1978. O tilito da base do Grupo Bambui na borda ocidental do Cráton do São Francisco. SBG, Núcleo Centro-Oeste, Boletim, 7-8:85-97.

Dardenne M.A., Freitas-Silva F.H., Nogueira G.M.S., Souza J.C.F. 1997. Depósitos de fosfato de Rocinha e Lagamar, Minas Gerais. In: Schobbenhaus C., Queiroz E.T., Coelho C.E.S. (coords.) Principais Depósitos Minerais do Brasil. DMPM/CPRM., IVc:113-122.

Dardenne M.A., Pimentel M.M., Alvarenga C.J.S. 2003. Provenance of conglomerates of the Bambuí, Jequitaí, Vazante and Ibiá Groups: Implications for the evolution of the Brasília Belt. In: SNET, 9. Búzios, Bol. de Resumos, p. 47-49.

Derby O. A. 1906. The Serra do Espinhaço, Brazil. The Journal of Geology, 14:374-401.

Evans D.A.D. 2000a. An updated geochronological synthesis of the world's Neoproterozoic glaciogenic deposits. In: Inter. Geol. Cong., 31, Rio de Janeiro, Brazil. Abstracts, CD-ROM.

Evans D.A.D. 2000b. Stratigraphic, geocronological and paleomagnetic constraints upon the Neoproterozoic climatic paradox. Am. J. Sci., 300:347-433.

Fernandes N.H., Brenner T.L., Carvalho S.G., Soares P.C., Morales N. 2000. Late Proterozoic iron and phosphatic 
formations in the Brasilia Colisional Belt, south Minas Gerais. Brazil. In: Intern. Geol. Cong., 31, Rio de Janeiro, Brazil, Abstracts, CD-ROM.

Figueiredo M.F., Babinski M., Alvarenga C.J.S., Pinho F.E.C. 2004. Diamictites overlaying Marionan-age carbonates of Araras Formation, Paraguay Belt, Brazil: evidence of a new glaciation?: In: Symp. on Neoproterozoic-Early Paleozoic Eventin SW-Gondwana, 1, Ext. Abstracts, IGCP Project 478, p.18.

Frimmel H.E. \& Fölling P.G. 2000. Neoproterozoic climate changes: the perspective from southern Africa. In: Intern. Geol. Cong., 31, Rio de Janeiro, Brazil, Abstracts, CDROM.

Gonzaga G.M. \& Tompkins L.A. 1991. Geologia do Diamante. In: Schobbenhaus C., Queiroz E.T., Coelho C.E.S. (coords.) Principais Depósitos Minerais do Brasil, IVa, DNPM/CPRM, p.53-116.

Gonzaga G.M. 2001. Glaciação Samburá (Neoproterozóico - Vendiano?) como possível agente transportador de diamantes no estado de Minas Gerais. Rev. Bras. Geoc., 31(4):597-604.

Gonzaga G.M. 2005. Glaciações do Neoproterozóico e reciclagem de diamantes, carbonados e safiras em Minas Gerais. In: SBG/Núcleo Minas Gerais, Simpósio Brasileiro de Geologia do Diamante, 4, Diamantina. Anais, Boletim $n^{\circ} 14$. p. 69-72.

Heilbron M., Pedrosa-Soares A.C., Campos Neto M.C., Silva L.C., Trouw R.A.J., Janasi V.A. 2004. Província Mantiqueira. In: Mantesso-Neto V., Bartorelli A., Carneiro C.D.R., Brito-Neves B.B. (org.) Geologia do Continente Sul-Americano: Evolução da obra de Fernando Flávio Marques de Almeida, Beca Prod. Culturais, p. 203-234.

Hoppe A., Schobbenhaus C.,Walde D.H.G. 1987. Precambrian iron-formation in Brazil. In: Appel P.W.U. \& LaBerge G.L. (eds.) Precambrian Iron-Formation. Theophastus Publications S.A. p. 347-390.

Karfunkel L.J., Dupont H., Hoppe A., Schobbenhaus C., Noce C.M., Peregovich B. 2000. The Neoproterozoic São Francisco Glaciation in central-eastern Brazil. In: Simposio sobre la Geología de Latinoamérica, 17, Anais, Universitat Stuttgart.

Karfunkel L.J., Martins M.S.M., Scholz R., McCandless. 2001. Diamonds from the Macaúbas river basin (MG, Brazil): Characteristics and possible source. Rev. Bras. Geoc. 31(4):445-456.

Kaufman A.J. 1998. Neoproterozoic chemostratigraphy: key events in earth history ordered by detailed intra and interbasinal correlation. In: Congress. Bras. Geol, 40, Belo Horizonte, SBG, Anais, v. 2.

Kaufman A.J., Olcott A., Sessions A., Brody K.B., Eigenbrode J., Cody G., Geboy N., Williams B., Corsetti F.A., Oliveira T.F., Azamy K., Misi A. 2005. Biomaker and stable isotope evidence for extreme productivity associated with Neoproterozoic Glaciation on the São Francisco Craton. In: Simpósio sobre o Cráton do São Francisco, 3, Salvador-BA, Short Papers, p. 274-278.

Leonardos H. 1940. Tilito metamórfico de Carandaí, Minas Gerais. In: Academia Brasileira de Ciências, Anais, 12:243-259.

Lima S.A.A., Martins-Neto M.A., Pedrosa-Soares A.C., Cor- dani U.G., Nutman A. 2003a. A Formação Salina na áreatipo, NE de Minas Gerais: uma proposta de revisão da estratigrafia da Faixa Araçuaí com base em evidências sedimentares, metamórficas e idades U-Pb SHRIMP. Rev. Bras. Geoc., 32(4):491-500.

Lima O.N.B., Morato L., Uhlein A. 2003b. Reconstituição de uma margem continental neoproterozóica no sul da Faixa Brasília: o Grupo Ibiá e a Formação Jequitaí. In: Simpósio de Geologia do Centro-Oeste, 8, Cuiabá, Brasil. Boletim de Resumos, p.32.

Misi A., Kaufman A. J., Veizer J., Powis K., Azmy K., Boggianni P.C., Gaucher C., Teixeira J.B.G., Sanches A.L., Iyer S.S.S. 2007. Chemostratigraphic Correlation of Neoproterozoic Successions in South America. Chem. Geol., 237:143-167.

Oliveira M.J.R., Grossi-Sad J.H., Romano A.W., Lobato L.M. 1997. Geologia da Folha Grão Mogol. Projeto Espinhaço, cap.7, COMIG. p. 611-713, CD-Rom.

Oliveira E.P., Carvalho M.J., Nascimento R.S., Araújo M.N., Dantas E., Basilici G., Bueno J.F., McNaughton N. 2005a. Evidence from detrital zircon geochronology and whole-rock SM-DN isotopes for off-craton provenance of clastic metasedimentaraty units of the Sergipano Belt, NE Brasil. In: Simpósio Nacional de Estudos Tectônicos. 10, Curitiba-PR, Boletim de Resumos Expandidos. p. 308-311.

Oliveira E.P, Carvalho M.J., Nascimento R.S., McNaughton N. 2005b. Evolution of the Neoproterozoic Sergipano Orogenic Belt, NE Brazil.: detrital zircon geochronology and SM-ND isotopes on metasedimentary rocks unravel part of the story. In: Simpósio sobre o Cráton do São Francisco, 3, Salvador-BA, Short Papers, 166-169p.

Pimentel M.M., Alvarenga J.S., Armstrong R. 2002. Proveniência da Formação Jequitaí, Brasil Central, com base em dados U-Pb SHRIMP em Zircões detríticos. In: Cong. Bras. Geol., 41, Anais, p. 503.

RADAMBRASIL. 1981. Levantamento de recursos naturais. Folha SD.22 Goiás. MME. Projeto RADAMBRASIL, Rio de Janeiro, Vol. 25, p. 636.

Ribeiro A., Paciullo F.V.P., Noce C.M., Valeriano C.M., Valença J.G., Ávila C.A., Trouw R.A.J., Silva M.A. 2003. Mapa Geológico - Folha de São João Del Rei (SF. 23$X$-C-11). Escala 1: 100.000. Projeto Sul de Minas, COMIG.

Schermerhorn L.J.G. 1983. Late Proterozoic glaciation in the ligth of $\mathrm{CO}_{2}$ depletion in the atmosphere. In: Medaris Jr. L.G., Byers C.W., Mickelson D.M., Shanks W.C. (eds.) Proterozoic Geology: Selected papers from an International Proterozoic Symposium. The Geological Society of America. Memoir, vol. 161, p.309-315.

Schobbenhaus C. \& Almeida Campos D. 1984. A evolução da Plataforma Sul-Americana no Brasil e suas principais concentrações minerais. In: Schobbenhaus C., Almeida Campos D., Derze G.R., Asmus H.E (coords.) Geologia do Brasil. DNPM, p. 9-53.

Seer H.J., Moraes L.C., Fogaça A.C.C. 1987. Faciologia e estruturação tectônica dos metassedimentos com diamictitos e jaspelitos da região de Lagoa Formosa-MG (Gr. Bambuí?). In: Simpósio de Geologia de Minas Gerais, 4, Anais, p. 199-213. 
Souza J.C.F. 1997. Litoestratigrafia e sedimentologia da Formação Vazante na região de Coromandel, $M G$. Dissertação de Mestrado, IG, Universidade de Brasília, Brasília, 75p.

Trompette R. 1994. Geology of Western Gondwana (2000500 Ma). Pan-African-Brasiliano aggregation of South America and Africa. A.A. Balkema, 350p.

Trouw R., Heilbron M., Ribeiro A., Paciullo F., Valeriano C.M., Almeida J.C.H., Tupinambá M., Andreis R.R. 2000. The central segment of the Ribeira Belt. In: Cordani U.G., Milani E.J., Thomaz Filho A., Campos D.A. (eds.) Tectonic Evolution of South America. In: Intern. Geol. Congress, 33, Rio de Janeiro, Brazil. p.287-310.

Uhlein A. 1991. Transição Cráton-faixa dobrada: Exemplo do Cráton do São Francisco e da Faixa Araçuai (Ciclo Brasiliano) no estado de Minas Gerais: Aspectos estratigráficos e estruturais. Tese Doutoramento, Universidade de São Paulo, p. 295.

Uhlein A., Alvarenga C.J.S., Trompette R., Egydio-Silva M., Cukrov N., Lima O.N.B. 2004. Glaciação neoproterozóica sobre o Cráton do São Francisco e faixas dobradas adjacentes. In: Mantesso-Neto V., Bartorelli A., Carneiro C.D.R., Brito-Neves B.B. (org.) Geologia do Continente Sul-Americano: Evolução da obra de Fernando Flávio Marques de Almeida, Beca Prod. Culturais, p. 539-553.

Vilela O.V., Pena C.A.T., Barsotti T.M., Jorge A.S. 1978. Prospecção das jazidas de minério de ferro dos municípios de Porteirinha, Rio Pardo de Minas, Riacho dos Machados e Grão Mogol, norte de Minas Gerais. In: Congresso Brasi- leiro de Geologia, 30, Recife, Anais, v. I, p.1914-1923.

Walde D.H.G. 1986. Geologia do ferro. In: Schobbenhaus C., Queiroz E.T., Coelho C.E.S. (coords.) Principais Depósitos Minerais do Brasil. DNPM/CPRM. v. II, p.3-6.

Walde D.H.G. \& Gonzaga G.M. 2005a. Neoproterozoic Glaciation in Brazil related to the Brasiliano Orogeny: discussion and model. In: Colloquium on Latin American Geosciences, 19 ${ }^{\text {th }}$, Potsdam, Abstract. p.138.

Walde D.H.G. \& Gonzaga G.M. 2005b. Evidence of a second glacial event during Neoproterozoic in Brazil: Selected examples. In: Simpósio Nacional de Estudos Tectônicos (SNET). 10, Curitiba. Boletim de Resumos Expandidos, p.294-296.

Walde D.H.G. \& Gonzaga G.M. 2005c. Glaciações do Neoproterozóico e o retrabalhamento de Diamantes na Faixa Araçuaí em Minas Gerais. In: SBG/Núcleo Minas Gerais, Simpósio Brasileiro de Geologia do Diamante, 4, Diamantina, Anais. Boletim 14, p. 54-57.

Walde D.H.G. \& Gonzaga G.M. 2005d. Evidence for mountain glaciation events in the Brasiliano Fold Belts surrounding the São Francisco Craton. In: Simpósio sobre o Cráton do São Francisco, 3, Salvador, Anais, p. 303305.

Manuscrito CSF-17

Submetido em 31 de maio de 2006 Aceito em 05 de novembro de 2007 